\title{
Support for Expert Estimations in Transportation Projects
}

\author{
O. Pastor, P. Novotný, J. Melechovský
}

This paper deals with risk analysis as a part of the financial assessment of transportation projects. Two approaches to risk assessment are discussed. A risk can be evaluated either directly in terms of the probabilistic distribution of the assessment criterion; or an indirect determination of the risk can be applied without constructing the probability distribution, but by determining the characteristic features of the project.

Keywords: decision-making, economic risk, direct determination of the project's risk, subjective probabilities.

Investment decision-making in a market economy is related to the uncertainty that ensues from the rapid and uncertain development of many factors. This uncertainty can also significantly influence the profitability of transportation projects and often leads to unexpected or undesirable results. Financial risk can generally be seen as a degree of risk that, if things go wrong, the financial outcome of a business activity will be worse than the anticipated outcome. Hence, risk analysis must form an integral part of any financial assessment of a transportation project. It forms part of a feasibility study of regional projects, and this is the basic document when the project is being defined. Risk analysis aims at finding the factors that most affect the risk of a given project and the importance of the particular risk. Subjective probabilities have to be applied at this preparatory stage of transportation project. The subjective probability is an expression of the degree of belief that an expert has in an uncertain proposition. It transforms his opinion and his subjective judgement into a quantitative expression.

Any risk assessment of transportation project involves determining (quantifying) the risk of the investigated variants of the project and preparing corrective measures that lead to an increased probability of success. Risk analysis can be schematically divided into the following stages: determining the functional dependency of a decision-making criterion on the influencing quantities, determining the risk factors and their relevance, determining the probabilities of risk factors, constructing the probability distribution of the considered decision-making criterion, and then assessing the risk of the project.

We can apply so-called subjective probabilities (Bayesian probabilities) to the probability assessment of risk situations at different stages of the risk analysis (a risk situation is a potential future situation having? an impact on the consequences of the considered decision-making variants known as "risk variants"). These probabilities are based on the presumption that every expert has a certain degree of faith in the occurrence of some phenomenon. In determining, these probabilities the expert will apply his or her knowledge, intuition, experience and various types of information. For example, the probability distribution of the risk factors is most often constructed on the basis of an expert analysis. In the case of discrete risk factors, the expert may either assess the probabilities of the individual values directly, or assess the parameters of some of the theoretical discrete probability distributions. However, risk factors with a discrete probability distribution occur rela- tively rarely. Risk factors with a continual probability distribution are more frequent. In the latter cases, it is suitable to use a simulation assessment by the Monte Carlo method to determine the probability distribution of a chosen criterion. Even in this scheme of application of the Monte Carlo method, subjective probabilities have to be used.

Therefore, within the development of tools for project risk analysis focused on practical application, the Department of Logistics and Transportation Processes at the Faculty of Transportation Sciences, Czech Technical University in Prague, also addresses the problem of constructing subjective probabilities with the aim to create and use a knowledge fund related to subjective probabilities.

An analysis of both direct and indirect methods for constructing subjective distribution of probabilities is being carried out for an individual decision-maker and also for a group of decision-makers. In the case of direct methods, experts express their persuasion numerically in the form of probability values, whereas in the case of indirect methods subjective probabilities are attained on the basis of an expert's behaviour in differentiating amongst multiple variants. A dialogue-type computer programme has been developed for the method of quantification of order; however tests showed that it was user-unfriendly.

Certain inadequacies of experts in determining subjective probabilities are examined, and the characteristics of the quality of subjective probabilities and factors that affect this quality are sought. The aim is to exert a favourable influence on the quality of project preparation. It was confirmed that experts tend to assess the distribution in the form of symmetrical distributions, and that the basic factors influencing the quality of subjective information are the influence of the expert (him or herself, his/her theoretical and practical knowledge and experience) and the methods applied in quantifying subjective information. Any piece of subjective information is always based on information that is accessible to an expert at a given moment. Different experts usually have different information sources, and therefore their probability assessments of a particular phenomenon may differ. Expressed by a mathematical model, let $\boldsymbol{P}(\boldsymbol{E}, \boldsymbol{I})$ be the subjective probability that an event $\boldsymbol{E}$ happens and $\boldsymbol{I}$ be the set of information on factors influencing the occurrence of the event. If the information base of different subjects is equalized, the subjective probabilities of event $\boldsymbol{E}$ should converge to a similar value. 
In conclusion, we would like to highlight that if subjective probabilities are used to quantify the risk of a project, we are not speaking about the real risk, but about the risk as it appears to the team of examiners. Risk analysis is not a technique in itself that can compensate for inadequacies in documents and in the judgement of the authors of a project. Its distinctive feature is that it attempts to combine exact methods and model tools with the knowledge and experience of researchers.

\section{Reference:}

[1] Pastor, O.: Project Development Cycle - Activities and Project Documentation. In: Automatizace. Czech Republic, Vol. 44 (2001), No. 12, p. 755-756. ISSN 0005-125X.

[2] Pastor, O.: Modelling of Economic Risks in Transport Projects. In: PRONT 2000. Pilsen: University of
West Bohemia, Czech Republic, 2001, p. 195-198. ISBN 80-7082-648-7.

Doc. Dr. Ing. Otto Pastor, CSc.

e-mail: pastor@fd.cvut.cz.

Ing. Petr Novotný

e-mail: petr.novotny@fd.cvut.cz

Ing. Jan Melechovský

e-mail: jan.melechov@seznam.cz

Department of Logistic and Transportation Processes

Czech Technical University in Prague

Faculty of Transportation Science

Horská 3

12803 Praha 2, Czech Republic 